\title{
Оценка эффективности экстракции малорастворимых примесей из водных растворов сверхкритическими флюидам в динамическом проточном режиме хромато-масс-спектрометрическим методом
}

\author{
(C) 2020 Паренаго О.О. ${ }^{1,2}$, Костенко М.О. ${ }^{1}$, Устинович К.Б. ${ }^{1}$, \\ Хесина 3.Б. ${ }^{3}$, Буряк А.К. ${ }^{3}$ \\ ${ }^{I}$ ФББУН Институт общей и неорганической химии РАН, Москва \\ ${ }^{2}$ Московский государственный университет им. М.В. Ломоносова, Москва \\ ${ }^{3}$ ФБУН Институт физической химии и электрохимии РАН, Москва
}

Поступила в редакцию 12.11.2020 г.

DOI: $10.17308 /$ sorpchrom.2020.20/3134

\begin{abstract}
Сверхкритическая флюидная экстракция (СФЭ) активно используется при выделении ценных компонентов или особо токсичных и радиоактивных веществ из различных природных источников, представляющих собой водные системы. Несмотря на активное изучение этого метода, возможности его изучены ещё недостаточно полно. В данной работе в качестве модельных объектов рассмотрены два малорастворимых вещества - трибутилфосфат (ТБФ) и метанитробензотрифторид (мета-нитротрифторметил-бензол, Ф-3), которые используются в технологиях выделения радионуклидов при переработке отработавшего ядерного топлива (ОЯТ). Целью этого исследования является оценка эффективности экстракционной очистки водных растворов, содержащих ТБФ и Ф-3, в динамических условиях на основании оценочного расчета и проверка его результатов в эксперименте на противоточной экстракционной колонне. В основу расчета положены данные о распределении примеси между водной и флюидной фазами, которые были получены в статических экспериментах. Концентрации веществ в исходных растворах (до проведения экстракции) определяли методом газовой хроматографии с массспектрометрическим детектированием (ГХ-МС) с помощью прибора SHIMADZU GCMS-TQ8040. Для хроматографического разделения использовали стандартную капиллярную колонку ZB-5MS $(60$ м $\times 0.25$ мм ×0.25 мкм). Этим же методом определяли остаточные концентрации ТФБ и Ф-3 в водных растворах после проведения экстракции. На основании данных по экстракции в статических условиях определены коэффициенты межфазного распределения (K) для ТБФ и Ф-3 между водной фазой и сверхкритическими (СК) флюидами - $\mathrm{CO}_{2}$ и фреоном R-23 $\left(\mathrm{CHF}_{3}\right)$ при $50^{\circ} \mathrm{C}$ и плотности флюидов около 0.4 г/ $\mathrm{cm}^{3}$. Показано, что в этих условиях $\mathrm{CK} \mathrm{CO}_{2}$ является более эффективным экстрагентом для выделения ТБФ и Ф-3 из водных растворов. Полученные величины К использованы для оценки эффективности экстракционной очистки водных растворов от ТБФ и Ф-3 в различных режимах. Работоспособность такой оценки продемонстрирована в экспериментах в противоточной экстракционной колонне (ПЭК). Показано, что во всех изученных парах «извлекаемый компонент - экстрагент» степень очистки в ПЭК может достигать 0.995 при умеренном расходе экстрагента.
\end{abstract}

Ключевые слова: коэффициент межфазного распределения, сверхкритический флюид, противоточная экстракционная колонна.

\section{Введение}

Экстракцией в широком смысле слова принято называть процессы извлечения одного или нескольких компонентов из растворов или твёрдых тел с помощью изби- 
рательных растворителей (экстрагентов) [1]. Экстракционные процессы классифицируются по тому, в какой фазе находятся растворяемое вещество и экстрагент, а также по способу организации процесса.

Метод сверхкритической флюидной экстракции (СФЭ) можно считать разновидностью флюид-флюидной экстракции, т.е. извлечения компонента, растворённого в одном флюиде путём его перевода в другую флюидную фазу, не смешивающуюся полностью с первой. Этому методу посвящено множество научных публикаций, в том числе большое число монографий, специализированных сборников и обзоров [2-7]. Основные его характеристики изучены как теоретически, так и экспериментально, многие процессы с его использованием достаточно хорошо отработаны практически и доведены до уровня реализованных технологий. Одним из относительно крупнотоннажных процессов, в котором используется СФЭ, является декофеинизация кофе и чая сверхкритическим (СК) $\mathrm{CO}_{2}[8,9]$. Однако именно этот процесс не относится к типу экстракции «флюид-флюид», поскольку исходным сырьём являются твёрдые вещества («матрицы») - кофейные зёрна и чайный лист.

Важно отметить, что именно выделение различных веществ из водных растворов и дисперсий наиболее эффективно может быть реализовано с использованием СФЭ, поскольку наиболее часто применяемые в ней флюиды - $\mathrm{CO}_{2}$, фреоны, пропан - имеют низкую растворимость в воде и сами достаточно мало растворяют воду. Кроме того, в рабочем диапазоне параметров, характерных для СФЭ, плотности водных растворов и флюидов-экстрагентов существенно различаются. По этим причинам не возникает сложностей с разделением экстракта и рафината (очищенной от растворённого вещества воды). В связи с этим СФЭ активно используется при выделении ценных компонентов из различных природных источников, представляющих собой водные системы (например, растительного сырья [10-14]), при определении содержания различных токсикантов в биологических средах [15], при очистке воды от загрязнений [16]. При технически более сложной организации процесса СФЭ используется не только для выделения, но и для фракционирования выделяемых из природных смесей веществ [17-19].

Особый интерес СФЭ представляет для выделения из водных сред особо токсичных и радиоактивных веществ, имеющих низкую собственную летучесть (давление насыщенных паров), поскольку при декомпресии (сбросе давления) СК флюида возможно понизить их концентрацию практически до нуля и максимально полно очистить экстрагент перед последующим сбросом или направлением его в систему рециркулирования. В данной работе в качестве модельных объектов рассмотрены два вещества - трибутилфосфат (ТБФ) и метанитробензотрифторид (мета-нитро-трифторметил-бензол, Ф-3), которые используются в технологиях выделения радионуклидов при переработке отработавшего ядерного топлива (ОЯТ).

ТБФ образует комплексы со многими катионами, включая многозарядные катионы лантаноидов, актиноидов, циркония и гафния, и применяется в качестве экстрагента для их извлечения из водных растворов [20,21]. Ф-3 используют в аналогичных процессах экстракции многозарядных катионов в качестве полярного разбавителя фосфорорганических комплексообразователей. Такому использованию ТБФ и Ф-3 способствует относительно низкая растворимость самих этих соединений в воде. Тем не менее, после экстракции компонентов ОЯТ водные растворы содержат остаточные количества органических веществ и требуют доочистки.

Целью этого исследования является оценка эффективности экстракционной очистки водных растворов, содержащих ТБФ и Ф-3, в динамических условиях на основании оценочного расчета и проверка его результатов в эксперименте на противоточной экстракционной колонне. В основу расчета положены данные о распределении 
примеси между водной и флюидной фазами, которые были получены в статических экспериментах.

В качестве экстрагентов использовались $\mathrm{CO}_{2}$ и фреон R-23 $\left(\mathrm{CHF}_{3}\right)$, отличающиеся в СК флюидном состоянии как физическими свойствами, так и растворяющей способностью по отношению к исследуемым органическим соединениям.

\section{Теоретическая часть}

\section{Описание метода расчета}

1. Распределение растворённого вещества между фазами. Возможность и эффективность экстракции типа «флюид-флюид» определяются, помимо несмешиваемости двух сред, величиной коэффициента межфазного распределения, который, строго говоря, выражает отношение концентраций растворённого вещества в двух флюидах при достижении равновесия:

$$
K=\frac{a_{2}}{a_{1}}
$$

где $\mathrm{a}_{1}$ и $\mathrm{a}_{2}$ - активности растворённого вещества в исходной среде и в экстрагенте, соответственно.

Уравнение (1) является выражением закона распределения Нернста [22]. В случае растворов с низкой концентрацией растворённого вещества, для которых активности можно заменить концентрациями, можно записать

$$
K=\frac{C_{2}}{C_{1}},
$$

где $\mathrm{C}_{1}$ и $\mathrm{C}_{2}$ - концентрации растворённого вещества в исходной среде и в экстрагенте, соответственно.

Физически величина К представляет собой отношение растворимостей распределяемого вещества в двух растворителях при одинаковых условиях. Растворимость в жидкости газообразного вещества в простейшем случае может описываться законом Генри

$$
C_{A, s}=\Gamma_{A} \cdot P_{A},
$$

где $\Gamma_{\mathrm{A}}$ - коэффициент Генри для вещества $\mathrm{A}, \mathrm{P}_{\mathrm{A}}$ - его парциальное давление.

Уравнение (2) показывает, что равновесная концентрация вещества А в жидкости пропорциональна его давлению. Закон Генри реализуется при относительно невысоких концентрациях растворённого вещества - в тех пределах, в которых оно само не изменяет в значительной степени физико-химические, а значит и растворяющие свойства жидкости. Отклонения от пропорциональности неизбежны в случаях концентрированных растворов.

Если речь идёт о растворимости жидкого вещества в другой жидкости, закон Генри может реализовываться в случае, если две жидкости являются не смешивающимися. В этом случае можно принять, что растворимость одной жидкости в другой подчиняется тому же закону Генри, но в правой части уравнения (2) присутствует давление насыщенного пара $\mathrm{P}_{\mathrm{A}, \mathrm{s}}$

$$
C_{A, S}=\Gamma_{A} \cdot P_{A, S}
$$

Следует также коротко рассмотреть вопрос о растворимости жидкости, имеющей малую летучесть (низкое значение $\mathrm{P}_{\mathrm{A}, \mathrm{s}}$ ), в СК флюиде. Если при испарении жидкости А в вакуум в замкнутом объёме равновесие устанавливается при достижении давления равного $\mathrm{P}_{\mathrm{A}, \mathrm{s}}$ и, соответственно, концентрации (в моль/л) в паровой фазе равной

$$
C_{A, V}=\frac{P_{A, S}}{R T}
$$


где $\mathrm{R}$ - универсальная газовая постоянная, T - абсолютная температура, пропущен блок текста!

Однако, в присутствии СК флюида давление и, соответственно, концентрация насыщения $\mathrm{C}^{*} \mathrm{~A}, \mathrm{~s}$ будет определяться интенсивностью межмолекулярных взаимодействий во флюидной фазе между молекулами двух веществ. По этой причине эта величина концентрации насыщения (условно говоря, растворимости жидкости в СК флюиде) должна зависеть не только от температуры (как давление и концентрация насыщенного пара), но и от плотности флюида. Если последнюю выразить также в единицах концентрации (например, в моль/дм³ ${ }^{3}$, то она, как правило, выше значения $\mathrm{C}_{\mathrm{A}, \mathrm{v}}$, определяемого выражением (4).

В случае распределения растворённой примеси А между двумя жидкостями, которые не только не смешиваются полностью друг с другом, но и весьма мало растворяются друг в друге (т.е. не модифицируют растворяющую способность по отношению к третьим веществам), величина К равна отношению коэффициентов Генри для двух растворителей

$$
K=\frac{\Gamma_{2, A}}{\Gamma_{1, A}}
$$

где $\Gamma_{1, \mathrm{~A}}$ и $\Gamma_{2, \mathrm{~A}}-$ коэффициенты Генри растворённого вещества в исходной среде и в экстрагенте, соответственно.

При переходе от распределения между двумя жидкостями к случаю экстракции из жидкости СК флюидом, мы можем воспользоваться аналогичным выражением с заменой величины $\Gamma_{2, \mathrm{~A}}$ на величину

$$
\Gamma_{2, A}^{*}=\frac{C_{A, S}^{*}}{P_{A, S}}
$$

Важно отметить, что в рассматриваемом случае соблюдения закона Генри (сильно разбавленные растворы) коэффициент $\mathrm{K}$ не зависит от концентраций $\mathrm{C}_{1}$ и $\mathrm{C}_{2}$. Соответственно, отношение концентраций вещества А в двух фазах не зависит ни от его суммарного количества в системе, ни от количества (массы, объёма) каждого из двух растворителей.

Эффективность экстракции, выражаемая степенью извлечения вещества в экстрагент, зависит от многих факторов, включающих: физико-химические свойства и характеристики фаз, между которыми перераспределяется растворённое вещество; способ организации процесса экстракции; интенсивность массообмена между фазами.

Подробный анализ влияния этих факторов на степень извлечения вещества можно найти в соответствующей литературе $[1,23]$. Краткое описание указанных зависимостей применительно к задачам данной работы приводится ниже.

$\mathrm{B}$ рассматриваемом случае независимости величины $\mathrm{K}$ от концентраций $\mathrm{C}_{1}$ и $\mathrm{C}_{2}$ степень извлечения вещества А из растворителя 1 (вода) в растворитель 2 (СК флюид) будет определяться выражением

$$
X=\frac{\left(M_{0}-M\right)}{M_{0}}=\frac{K \cdot V_{2}}{\left(V_{1}+K \cdot V_{2}\right)}
$$

где $\mathrm{X}$ - степень извлечения вещества из водной фазы, $\mathrm{M}_{0}, \mathrm{M}$ - исходное и текущее (конечное) массовое содержание вещества в водной фазе, соответственно, $\mathrm{V}_{1}$ - объём водной фазы, $\mathrm{V}_{2}$ - объём фазы экстрагента.

Выражение (6) позволяет оценить эффективность равновесного извлечения вещества А из растворителя 1 растворителем 2 при известной величине К. На рис. 1 приведены зависимости величины $\mathrm{X}$ от соотношения объёмов очищаемого раствора $\left(\mathrm{V}_{1}\right)$ и экстрагента $\left(\mathrm{V}_{2}\right)$ при различных значениях $\mathrm{K}$ (рис. 1a) и от $\mathrm{K}$ при различных отношениях $\mathrm{V}_{2} / \mathrm{V}_{1}$ (рис. 1б). 

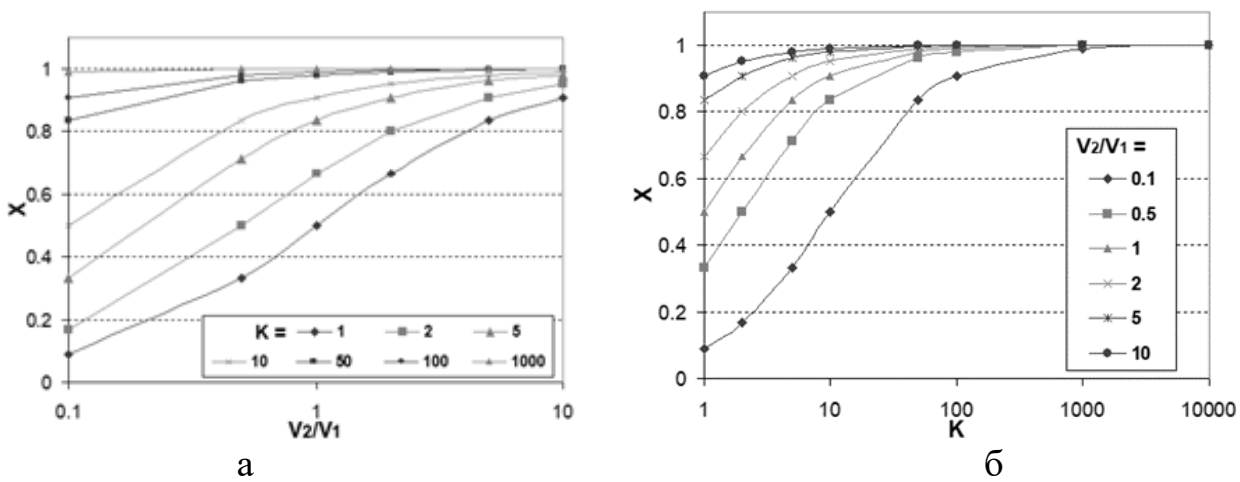

Рис. 1. Зависимость эффективности экстракции $\mathrm{X}$ от отношения объёмов $\mathrm{V}_{2} / \mathrm{V}_{1}$ при постоянных величинах $\mathrm{K}$ (а) и от величины $\mathrm{K}$ при постоянных отношениях $\mathrm{V}_{2} / \mathrm{V}_{1}$.

Fig. 1. Dependence of the extraction efficiency $X$ on the volume ratio $V_{2} / V_{1}$ at constant $\mathrm{K}$ values (a) and on the $\mathrm{K}$ value at constant $\mathrm{V}_{2} / \mathrm{V}_{1}$ ratios.

Наконец, при заданной величине К достижение заданной степени выделения примеси $\mathrm{X}$ возможно при отношении $\mathrm{V}_{2} / \mathrm{V}_{1}$ не ниже величины, задаваемой выражением

$$
\frac{V_{2}}{V_{1}}=\frac{X}{K(1-X)}
$$

Таким образом, при соблюдении сформулированных допущений, эффективность выделения определяется величиной коэффициента $\mathrm{K}$ и соотношением объёмов фаз исходного раствора и экстрагента. Однако величина $\mathrm{X}$, определяемая уравнением (7), является предельной, т.е. достигаемой при установлении равновесного распределения растворённого вещества между двумя фазами. Поскольку в данной работе стоит задача экстракционного выделения из водных растворов примесей органических веществ, имеющих низкую растворимость в воде, речь идёт о растворах с низкими концентрациями, это означает, что с большой вероятностью все выше сформулированные допущения реализуются и приведённые соотношения можно использовать для описания и анализа процесса экстракции.

2. Организация процесса экстракции. Сказанное в предыдущем разделе относится к однократной равновесной экстракции. Как следует из данных рис. 1а-б и выражения (8), достижение высоких степеней очистки водных растворов от примесей малорастворимых органических веществ возможно при использовании экстрагентов, в которых эти вещества растворяются существенно лучше (высокие значения К) и при использовании значительных объёмов растворителей. При этом имеется ряд приёмов, позволяющих снизить объём используемого экстрагента без снижения эффективности выделения. Ниже рассматриваются некоторые из таких приёмов.

\section{1. Многостадийная экстракция}

Даже при относительно невысоких значениях К добиться высокой степени извлечения примеси можно с использованием многостадийной экстракции. Если на единичной стадии достигается степень очистки, определяемая выражением (7), то после $\mathrm{n}$ таких стадий достигается степень очистки

$$
X_{n}=1-\left(1-\frac{K V_{2}}{V_{1}+K V_{2}}\right)^{n}
$$

Построенные с использованием выражения (9) кривые, представленные на рис. 2a-б, показывают, что даже при относительно невысокой степени однократного выделения, можно достичь высоких степеней очистки раствора от примеси с использованием многократной (многостадийной) экстракции. 

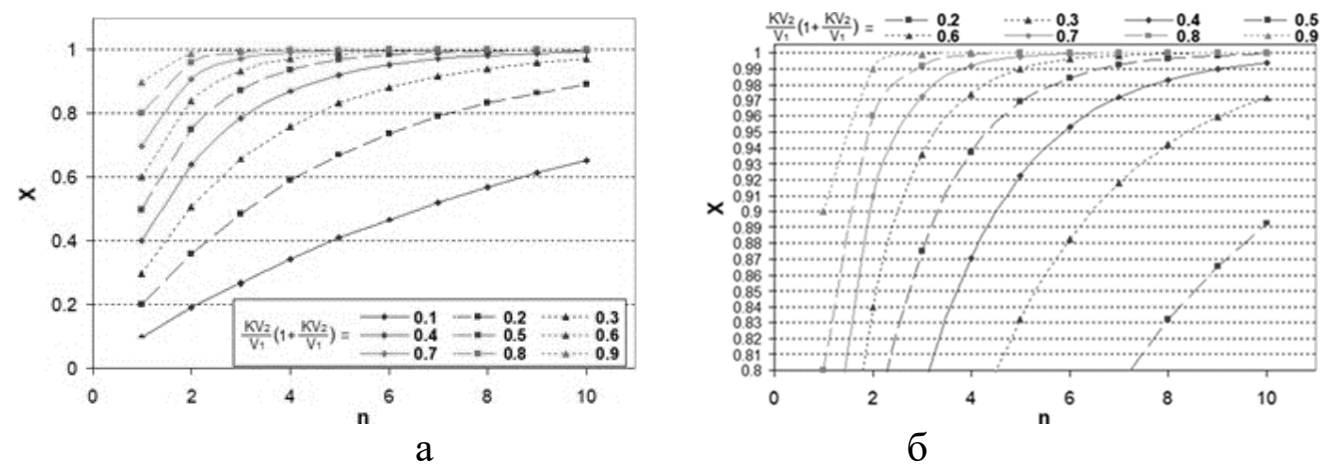

Рис. 2. Зависимость эффективности экстракции X от числа стадий при различных отношениях $\mathrm{V}_{2} / \mathrm{V}_{1}$.

Fig. 2. Dependence of the extraction efficiency $X$ on the number of stages at various $\mathrm{V}_{2} / \mathrm{V}_{1}$ ratios.

Недостатком описанного метода является существенное увеличение расхода экстрагента. Даже при использовании такого относительно недорогого экстрагента, как $\mathrm{CK} \mathrm{CO}_{2}$, очистка которого от органических примесей не является технически сложной, увеличение расхода может привести к дополнительным издержкам за счёт увеличения металлоёмкости оборудования и возрастания потребления энергии.

\section{2. Дробная экстракция}

Под дробной экстракцией (её ещё называют перекрёстной) будем понимать разделение общего объёма экстрагента на несколько порций и использование этих малых порций последовательно. Такой подход позволяет увеличить эффективность выделения примеси и очистки растворителя (в данном случае - воды) при сохранении суммарного заданного объёма экстрагента. Если объёмы $\mathrm{m}$ порций равны и в каждом последовательном шаге достигается равновесие, то суммарная степень очистки определяется выражением

$$
X_{n}=1-\left(1-\frac{K V_{2}}{m V_{1}+K V_{2}}\right)^{m}=1-\left(1-\frac{K \frac{V_{2}}{V_{1}}}{m+K \frac{V_{2}}{V_{1}}}\right)^{m}
$$

Приведённые на рис. За-б кривые, построенные на основании выражения (10) показывают, что даже при относительно невысоких значениях К этот метод позволяет достигать существенного повышения эффективности выделения примеси (в данном случае - очистки воды). Так уже при $\mathrm{K}=10$ разбиение суммарного объёма экстрагента на 5 порций приводит к возрастанию суммарной величины X с $\sim 0.91$ до $\sim 0.996$, или к снижению концентрации примеси в воде в 22 раза.
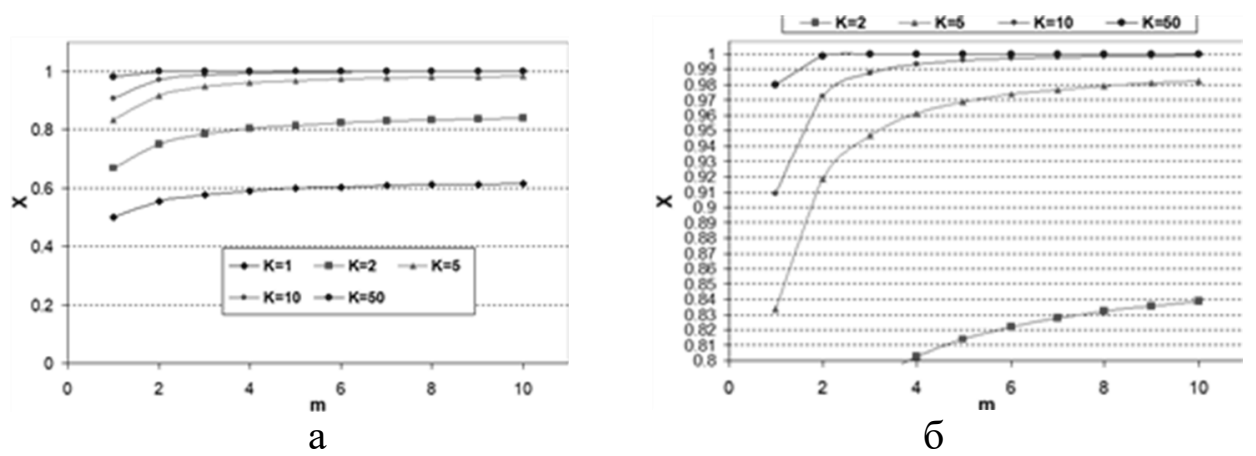

Рис. 3. Зависимость эффективности экстракции $\mathrm{X}$ от числа порций экстрагента при постоянном отношении $\mathrm{V}_{2} / \mathrm{V}_{1}=1$ и при различных значениях $\mathrm{K}$.

Fig. 3. Dependence of the extraction efficiency $\mathrm{X}$ on the number of portions of the extractant at a constant ratio $V_{2} / V_{1}=1$ and at different $\mathrm{K}$ values. 
Также следует указать, что случай $\mathrm{m} \rightarrow \infty$ соответствует медленной продувке раствора фиксированным количеством СК флюида при интенсивном перемешивании.

3. Непрерывная экстракция в потоке

Дальнейшее усложнение и одновременное повышение производительности экстракционного процесса связано с использованием проточных систем, отличающимися различными параметрами, в первую очередь - направлениями потоков очищаемого раствора и экстрагента. Наиболее простые варианты реализации экстракции в потоке являются прямоточный (два потока движутся в одном направлении) и противоточный (потоки движутся в противоположных направлениях). В первом случае возможно достижение степени извлечения примеси, соответствующей однократной равновесной экстракции при соотношении скоростей потока очищаемого раствора $\left(\mathrm{W}_{1}\right)$ и экстрагента $\left(\mathrm{W}_{2}\right)$

$$
\frac{W_{2}}{W_{1}}=\frac{V_{2}}{V_{1}}
$$

причём приближение к равновесию достигается при снижении скоростей потоков. Преимуществом такого способа организации процесса является его непрерывность. Однако увеличение степени извлечения примеси достигается при снижении производительности.

Интенсификации процесса удаётся достигнуть при организации противотока. В этом случае в каждом сечении аппарата перпендикулярном потокам фаз процесс протекает при максимальной величине движущей силы. Под ней понимают разницу между равновесной и текущей концентрациями примеси в экстрагенте при данном суммарном её содержании в соответствии с выражением (7). Хотя уже из этого утверждения следует, что глобальное равновесие между двумя фазами не достигается, эффективность такого выделения может быть очень высокой, поскольку этот способ является в некотором роде проточным аналогом дробной экстракции при $\mathrm{m} \rightarrow \infty$ (см. выше). Если в сечении аппарата на входе исходного раствора максимальны концентрации примеси в обеих фазах, то в противоположном сечении (на входе экстрагента и на выходе рафината) они минимальны. При этом из подлежащего очистке растворителя с минимальным содержанием примеси производится её экстракция практически чистым экстрагентом.

Как и во всех предыдущих рассмотренных случаях, степень извлечения примеси возрастает при увеличении отношения $\mathrm{V}_{2} / \mathrm{V}_{1}$ (в данном случае $-\mathrm{W}_{2} / \mathrm{W}_{1}$ ). Степень приближения к равновесию растёт при увеличении суммарного времени контакта между фазами, т.е. при снижении абсолютных величин $\mathrm{W}_{2}$ и $\mathrm{W}_{1}$ при заданном объёме аппарата или при увеличении объёма аппарата.

Для каждого аппарата можно определить эффективную величину параметра m, соответствующую числу условных порций, на которое разбивается объём экстрагента, приходящийся на единицу объёма очищаемой фазы. По аналогии с ректификацией, её можно назвать «числом теоретических тарелок» для экстракционного выделения. В случае СФЭ эта величина может существенно варьироваться при изменении параметров (в первую очередь - температуры и давления) ввиду зависимости динамических характеристик межфазного переноса в паре «очищаемая жидкость - экстрагент» от физического состояния СК флюида. Скорость межфазного обмена может возрастать при его интенсификации, например, за счёт увеличения поверхности контакта фаз и/или организации перемешивания. 


\section{Экспериментальная часть}

В работе использованы растворы ТБФ и Ф-3 в дистиллированной воде. Образцы ТБФ и Ф-3 предоставлены Акционерным обществом «Высокотехнологический научно-исследовательский институт неорганических материалов имени академика А.А. Бочвара» (Москва, РФ). В качестве экстрагента использовали диоксид углерода марки пищевой (99.8\%) производства ОАО «Линде-Газ Рус» (Балашиха, М/о), ГОСТ № 8050-85, Двуокись углерода высокой чистоты марка «4.5», ТУ20.1112-01600153318-2019 (АО «Московский газоперерабатывающий завод, Москва), Хладагент R-23 (Изготовитель - GALCO S/F/CLIMALIFE, Бельгия, Сертификат соответствия № РОСС ВЕ.АД.83НО1328).

Концентрации веществ в исходных растворах (до проведения экстракции) определяли методом газовой хроматографии с масс-спектрометрическим детектированием (ГХ-МС) с помощью прибора SHIMADZU GCMS-TQ8040. Для хроматографического разделения использовали стандартную капиллярную колонку ZB-5MS $(60 \mathrm{M} \times 0.25$ мм $\times 0.25$ мкм). Этим же методом определяли остаточные концентрации ТФБ и Ф-3 в водных растворах после проведения экстракции.

Перед проведением анализа проводили предварительную экстракцию органических примесей из водных растворов в соответствии с процедурами, описанными в литературе [24]. Для этого к $0.5 \mathrm{~cm}^{3}$ исследуемого водного раствора добавляли $0.5 \mathrm{~cm}^{3}$ гексана, интенсивно встряхивали в течение 5 минут, после расслоения раствора на две фазы 100 мкл верхнего гексаного слоя с экстрагированными аналитами отбирали в стеклянную виалу, которую помещали в систему ввода проб хромато-масс-спектрометра.

Анализ проводили в следующем режиме: объем вводимой пробы 4 мкл; начальная температура колонки $60^{\circ} \mathrm{C}$; температура инжектора $250^{\circ} \mathrm{C}$; ввод пробы с делением потока в отношении 1:20; газ-носитель - гелий; скорость потока газа-носителя $2.06 \mathrm{~cm}^{3} / \mathrm{мин;} \mathrm{режим} \mathrm{хроматографирования:} \mathrm{с} 0$ по 1 мин изотерма $60^{\circ} \mathrm{C}$, далее нагрев от 60 до $150^{\circ} \mathrm{C}$ со скоростью $70^{\circ} \mathrm{C} /$ мин, далее нагрев от 150 до $305^{\circ} \mathrm{C}$ со скоростью $20^{\circ} \mathrm{C} /$ мин, далее 5 мин изотерма $305^{\circ} \mathrm{C}$; ионизация электронами; температура ионного источника $150^{\circ} \mathrm{C}$; регистрация хроматограммы по полному ионному току; диапазон сканирования массовых чисел $\mathrm{m} / \mathrm{z}=45-600$; время сканирования 5 сек.

Идентификацию веществ проводили путём сравнения зарегистрированных масс-спектров с библиотекой (базой данных) масс-спектров NIST 2017. Их концентрации определяли методом внешнего стандарта. Примеры полученных хроматограмм и масс-спектров, а также сравнение зарегистрированных масс-спектров с массспектрами из библиотеки NIST 2017, представлены на рис. 4-7.

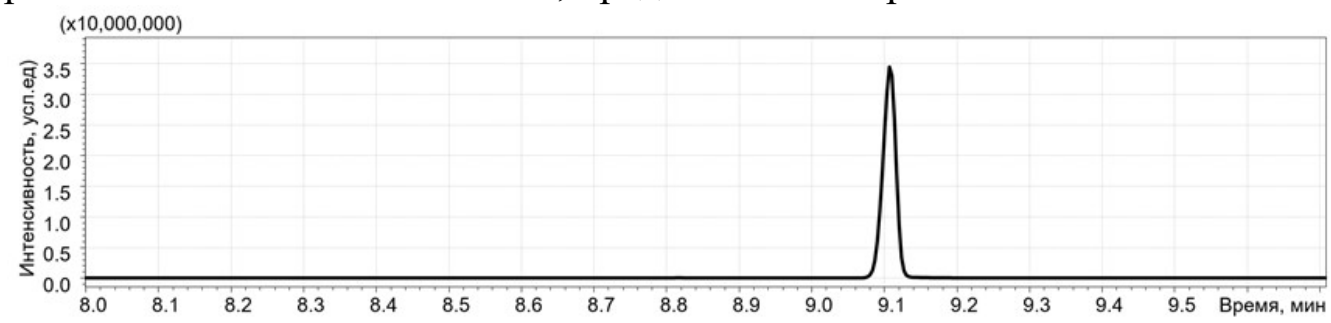

Рис. 4. Фрагмент хроматограммы, полученной в режиме сканирования по полному ионному току, стандартного образца водного раствора ТБФ; время удерживания вещества -9.11 мин.

Fig. 4. Fragment of a chromatogram obtained in the scan mode for total ion current of a standard sample of TBP aqueous solution; the retention time of the substance is $9.11 \mathrm{~min}$. 


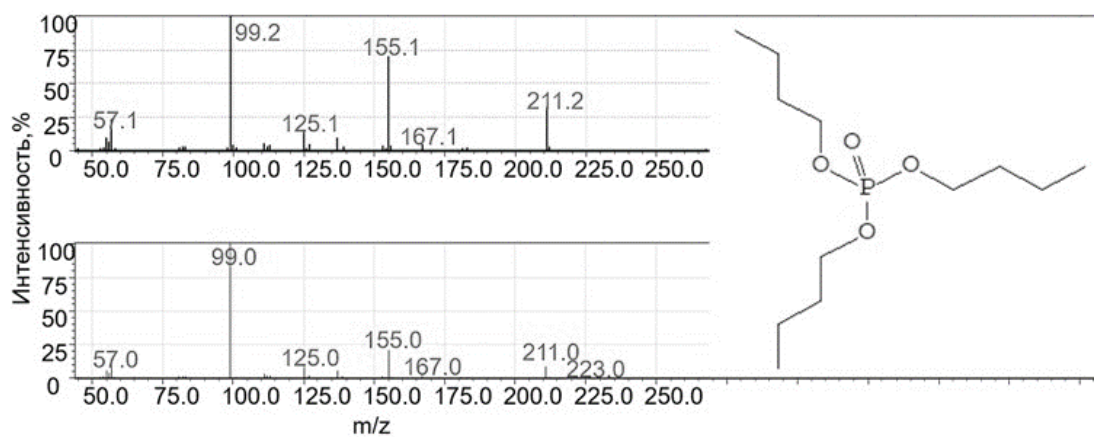

Рис. 5. Масс-спектры вещества с временем удерживания 9.11 мин. (вверху - зарегистрированный масс-спектр, внизу - библиотечный), коэффициент идентичности 0.92 .

Fig. 5. Mass spectra of a substance with a retention time of $9.11 \mathrm{~min}$. (top - registered mass spectrum, bottom - library mass spectrum), identity coefficient is 0.92 .

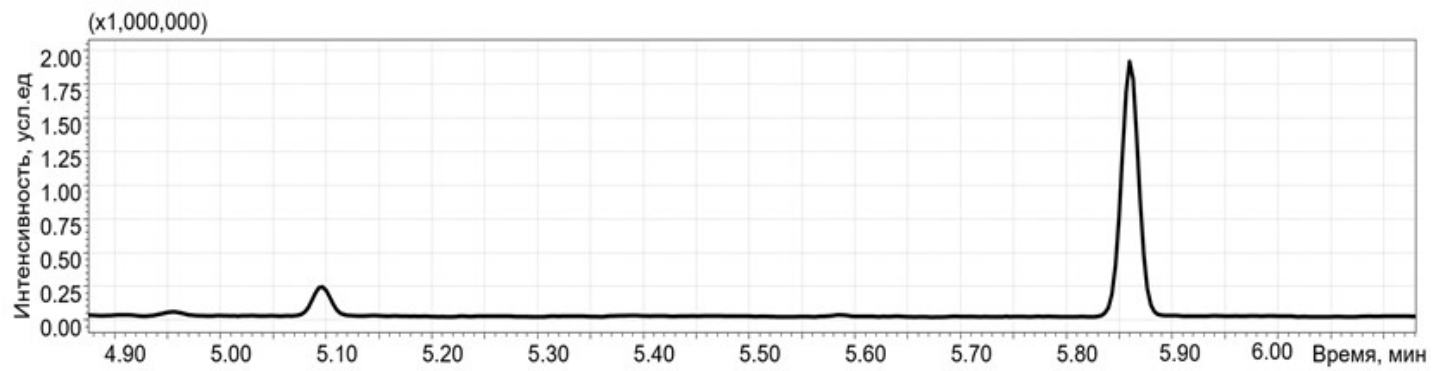

Рис. 6. Фрагмент хроматограммы, полученной в режиме сканирования по полному ионному току, образца водного раствора Ф-3. Время удерживания вещества -5.86 мин.

Fig. 6. Fragment of a chromatogram obtained in scan mode for the total ion current of a sample of F-3 aqueous solution. The retention time of the substance is 5.86 minutes.

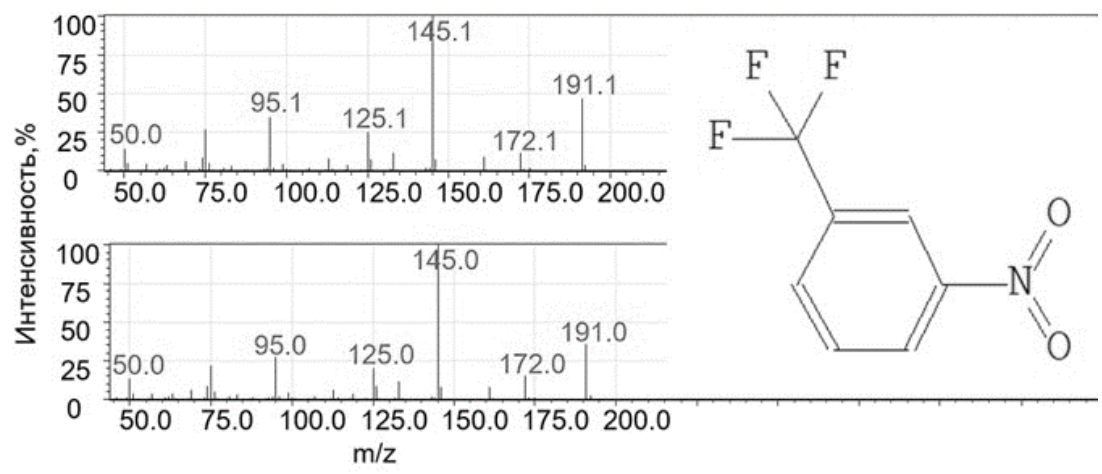

Рис. 7. Масс-спектры вещества с временем удерживания 5.86 мин. (вверху - зарегистрированный масс-спектр, внизу - библиотечный), коэффициент идентичности 0.92 .

Fig. 7. Mass spectra of a substance with a retention time of $5.86 \mathrm{~min}$. (top - registered mass spectrum, bottom - library mass spectrum), identity coefficient is 0.92 .

Эксперименты по экстракции органических веществ из водных растворов в статических условиях для определения величин коэффициентов К проводили на установке, схема которой представлена на рис. 8. Исходный водный раствор в количестве $10 \mathrm{~cm}^{3}$ загружали автоматической пипеткой в стальной автоклав высокого давления объёмом $20 \mathrm{~cm}^{3}$ (1). После герметизации автоклав присоединяли к насосу подачи флюида (2) Supercritical-24 (SSI Teledyne, США) через игольчатый вентиль (3) (НiP, США). Термостатирование автоклава осуществляли погружением в жидкостной термостат (4), собранный на основе погружного блока M02 (Термэкс, Томск) и заполненный во- 
дой. Температура во всех опытах составляла $50^{\circ} \mathrm{C}$. Все соединения в экспериментальной установке были выполнены из стального капилляра внешним диаметром 1/16", внутренним диаметром 0.5 мм. Автоклав помещали в термостат и выдерживали в течение 5 минут для достижения температурного равновесия, после чего насосом подавали экстрагент (диоксид углерода или трифторметан) до достижения необходимого давления. В работе проводились опыты с использованием диоксида углерода при 10 МПа и трифторметана при 7 МПа. После набора давления систему выдерживали в термостате в течение 30 минут при активном перемешивании среды встряхиванием при помощи внутреннего якоря. После этого вентиль (3) перекрывали, извлекали автоклав из термостата и отсоединяли от насоса. Отбор пробы водной фазы объёмом 2$3 \mathrm{~cm}^{3}$ из автоклава проводили через вентиль (3). После сброса давления автоклав промывали дистиллированной водой и ацетоном и высушивали перед проведением следующего эксперимента.

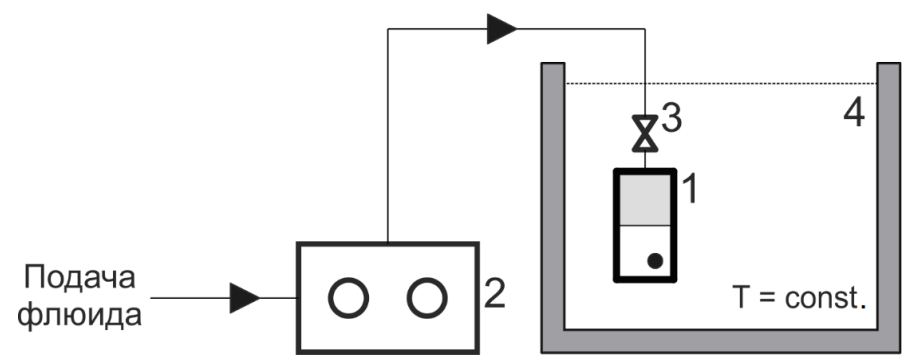

Рис. 8. Схема установки для проведения экстракции в статических условиях: 1 - автоклав, 2 - насос, 3 - игольчатый вентиль, 4 -жидкостной термостат.

Fig. 8. Installation diagram for extraction under static conditions: 1 - autoclave, 2 - pump, 3 - needle valve, 4 - liquid thermostat.

Исходя из предположения о малой взаимной растворимости воды и используемых в работе флюидов, массовые количества $\mathrm{CO}_{2}$, и $\mathrm{CHF}_{3}$, подаваемые в автоклав, можно вычислить исходя из известного объёма флюидной фазы $\left(10 \mathrm{~cm}^{3}\right)$ и значений плотности, определяемых по диаграммам «давление - плотность», приведённым на рис. 9 и построенным по данным [25]. Для $\mathrm{CO}_{2}$ при 10 МПа и $\mathrm{CHF}_{3}$ при 7 МПа эти количества составляют 3.8 и 4.1 г, соответственно г.

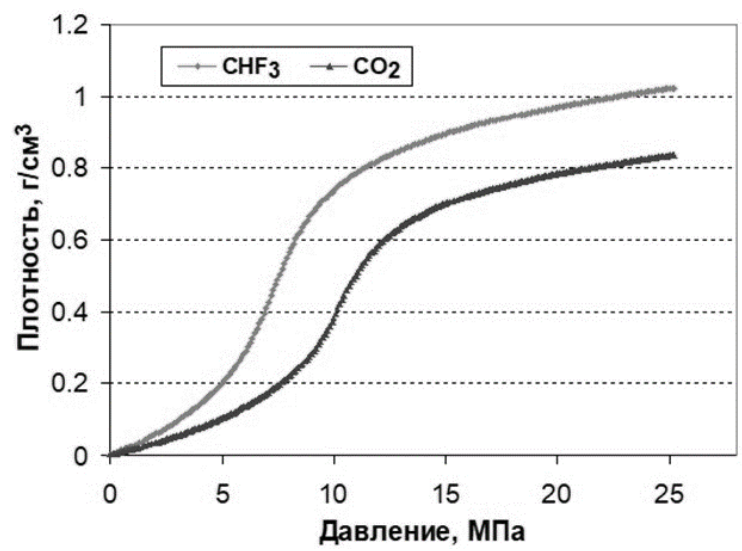

Рис. 9. Зависимость плотности флюида от давления при $50^{\circ} \mathrm{C}$ для $\mathrm{CO}_{2}$, и $\mathrm{CHF}_{3}$ по данным[25].

Fig. 9. Pressure dependence of fluid density at $50^{\circ} \mathrm{C}$ for $\mathrm{CO}_{2}$, and $\mathrm{CHF}_{3}$ according to [25].

Эксперименты по экстракции органических веществ из водных растворов в противоточной колонне проводили на установке, схема которой представлена на рис. 10. 
Для выделения растворённого вещества водный раствор, находящийся в сосуде (1), подаётся при помощи насоса (2) через запорные вентили (3) или (4), соответственно, в верхний или средний входные порты экстракционной колонны (5). В нижний входной порт противоточной экстракционной колонны (5) через вентиль (6) подаётся СК экстрагент, находящийся в баллоне (7). Экстрагент предварительно ожидается охлаждением в теплообменнике (8) и через расходомер (9) насосом (10) подаётся при заданном рабочем давлении в теплообменник (11), в котором нагревается до рабочей температуры и переводится в СК состояние. В результате массообмена (экстракции растворённого вещества) между водным раствором и СК экстрагентом образуется очищенная водная фаза (рафинат), которая выводится из колонны (5) через сливной вентиль (12). Поток СК экстрагента, содержащий выделенное из водной фазы органическое вещество, через выпускной вентиль (13) и автоматический регулятор обратного давления (14) поступает в циклонный сепаратор (15), в котором из экстрагента при сбросе давления и снижении растворяющей способности флюида (CO2 или фреона) выделяется растворённое вещество. Условия разделения в сепараторе (15) задаются при помощи ручного регулятора обратного давления (16). Собранное в сепараторе органическое вещество выводится из него при помощи выпускного крана (17).

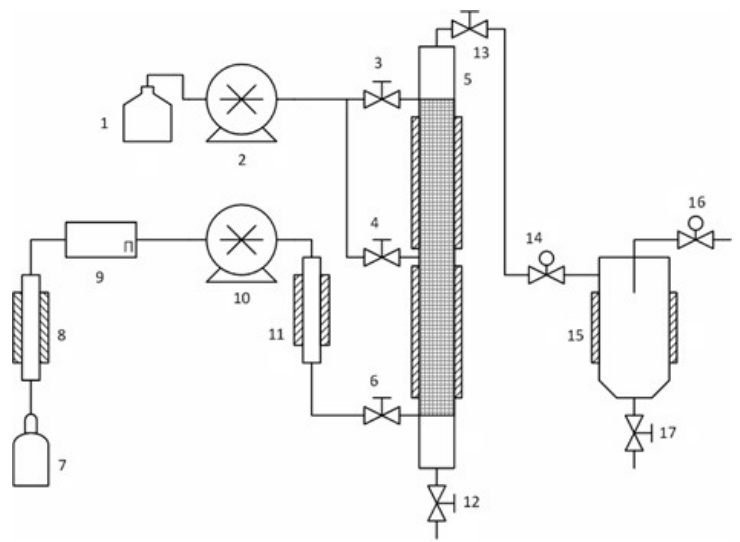

Рис. 10. Схема экспериментальной установки с противоточной экстракционной колонной: 1 - сосуд с исходным водным раствором, 2 - насос подачи водного раствора, 3 - вентиль подачи водного раствора в верхний входной порт, 4 - вентиль подачи водного раствора в средний входной порт, 5 - противоточная экстракционная колонна, 6 - вентиль подачи СК экстрагента в нижний входной порт, 7 - баллон с экстрагентом (фреоном или $\left.\mathrm{CO}_{2}\right), 8$ - предварительный теплообменник охлаждения экстрагента, 9 - расходомер, 10 - жидкостной насос подачи экстрагента, 11 - теплообменник нагрева экстрагента, 12 - вентиль слива рафината, 13 - вентиль выпуска потока экстрагента, 14 - автоматический регулятор обратного давления, 15 - циклонный сепаратор, 16 - ручной регулятор обратного давления, 17 - вентиль слива экстракта (выпускной кран).

Fig. 10. Scheme of the experimental equipment with a counter-current extraction column: 1 - vessel with an initial aqueous solution, 2 - pump for supplying an aqueous solution, 3 - valve for supplying an aqueous solution to the upper inlet port, 4 - valve for supplying an aqueous solution to the middle inlet port, 5 - counter-current extraction column, 6 - valve for supplying an SC extractant to lower inlet port, 7 - cylinder with an extractant $\left(\right.$ Freon or $\left.\mathrm{CO}_{2}\right), 8$ - preliminary heat exchanger for cooling the extractant, 9 - flow detector, 10 - fluid pump for supplying the extractant, 11 - heat exchanger for heating the extractant, 12 - raffinate drain valve, 13 - extractant flow outlet valve, 14 - automatic back pressure regulator, 15 - cyclone separator , 16 - manual back pressure regulator, 17 - extract drain valve (discharge cock).

Был определён следующий порядок работы: после прогрева до заданной температуры колонна заполняется $\mathrm{CO}_{2}$ до заданного давления и подаётся водный раствор органического вещества. Каждые 10 мин. накопившаяся в нижнем отделе колонны в 
количестве $100 \mathrm{~cm}^{3}$ рафинат сливается в приёмник. Первая порция не учитывается как нестационарная. Следующие три порции $\left(300 \mathrm{~cm}^{3}\right)$ соединяются для усреднения результатов. После окончания каждого эксперимента тракт подачи водного раствора и колонна промываются потоком чистой воды в течение 20 мин.

В работе использована противоточная экстракционная колонна производства Waters Corp. (США), обладающая следующими характеристиками: максимальное рабочее давление флюида - 600 бар, максимальная рабочая температура $-150^{\circ} \mathrm{C}$, объем колонны $-1500 \mathrm{~cm}^{3}$, количество экстракционных секций -2 ; количество зон температурного контроля -4 .

Основными параметрами, определяющими режим и эффективность работы противоточной экстракционной колонны, являются: тип используемого экстрагента; температура; давление СК флюида (экстрагента); расходы (объёмные скорости подачи) водного раствора и экстрагента (СК флюида); время контакта между водным раствором и экстрагентом (СК флюидом), регулируемое местом ввода водного раствора в колонну (верхний или средний входной порт).

Интервал возможных соотношений потоков водного раствора СК флюида был определен в предварительных экспериментах на конкретной колонне исходя из следующих условий: водный раствор не должен выноситься потоком флюида в сепаратор с экстрактом; рафинат (очищенная вода) не должен застаиваться в колонне - должен поступать в приемную емкость.

Для модельного эксперимента были выбраны следующие величины скоростей подачи: водный раствор - $10 \mathrm{~cm}^{3} /$ мин, экстрагент- 20 г/мин, или в единицах объёмной скорости подачи 52.5 и $48.6 \mathrm{~cm}^{3} /$ мин. СК $\mathrm{CO}_{2}$ и $\mathrm{CHF}_{3}$ при давлениях 10 и $7 \mathrm{MПа,} \mathrm{со-}$ ответственно. Таким образом, величина отношения $\mathrm{W}_{2} / \mathrm{W}_{1}$, которая является одним из параметров, определяющих степень очистки водного раствора от органической примеси, составляла 5.25 и 4.86.

Величины температуры и рабочего давления экстрагента $\left(\mathrm{CK} \mathrm{CO}_{2}\right)$ в статических и динамических экспериментах были одинаковыми: $+50^{\circ} \mathrm{C}$ и $10 \mathrm{MПа.} \mathrm{Поскольку}$ в задачи данной работы не входила оптимизация процесса экстракции, эти параметры не варьировали.

\section{Обсуждение результатов}

Данные, полученные при анализе методом ГХ-МС исходных и обработанных СК флюидами-экстрагентами водных растворов органических веществ в статических условиях представлены в таблице 1.

Таблица 1. Экстракция ТБФ и Ф-3 СК флюидами и оценка параметров экстракции при различных способах её реализации.

Table 1. Extraction of TBP and F-3 SC with fluids and estimation of extraction parameters with various methods of implementation.

\begin{tabular}{|c|c|c|c|c|c|c|c|}
\hline № & Вещество & $\begin{array}{c}\text { Обработка } \\
\left(50^{\circ} \mathrm{C}\right)\end{array}$ & $\begin{array}{l}\text { Конц-я, } \\
\text { мг/дм }{ }^{3}\end{array}$ & $\mathrm{~K}^{*}$ & $\begin{array}{l}\mathrm{V}_{2} / \mathrm{V}_{1} \\
(\mathrm{n}=1)\end{array}$ & $\begin{array}{c}\mathrm{n} \\
\left(\mathrm{V}_{2} / \mathrm{V}_{1}=1\right)\end{array}$ & $\mathrm{m}^{* *}$ \\
\hline 1 & \multirow{3}{*}{ ТБФ } & Исходный & 5.68 & & & & \\
\hline 2 & & $\mathrm{CO}_{2}, 10 \mathrm{MПа}$ & 0.13 & 43 & 4.6 & $<2$ & 1 \\
\hline 3 & & $\mathrm{CHF}_{3}, 7 \mathrm{MПа}$ & 2.19 & 1.6 & 124 & $\sim 6$ & $\sim 7$ \\
\hline 4 & \multirow{3}{*}{$\Phi-3$} & Исходный & 4.25 & & & & \\
\hline 5 & & $\mathrm{CO}_{2}, 10 \mathrm{M \Pi а}$ & 0.25 & 16 & 12.4 & 2 & $<2$ \\
\hline 6 & & $\mathrm{CHF}_{3}, 7 \mathrm{MПа}$ & 0.48 & 7.9 & 25 & $\sim 3$ & $\sim 2$ \\
\hline
\end{tabular}

* - величина коэффициента межфазного распределения; ** - для условий экстракции в противоточной колонне при $\mathrm{W}_{2} / \mathrm{W}_{1}=5.25$ для $\mathrm{CO}_{2}$ и 4.86 для $\mathrm{CHF}_{3}$. 
Полученные величины К позволяют определить объёмы СК экстрагентов, необходимые для достижения требуемой степени очистки водных растворов. В частности, в Таблице приведены необходимые для достижения величины $\mathrm{X}=0.995$ величины параметров для различных способов организации СФЭ:

а. однократная экстракция; по уравнению (8): $\left(\mathrm{V}_{2} / \mathrm{V}_{1}\right)=0.995 /(0.005 \times \mathrm{K})$

б. многостадийная экстракция при $\mathrm{V}_{2}=\mathrm{V}_{1}$; по уравнению (9): $\mathrm{n} \approx \lg (1-0.995) / \lg [1-\mathrm{K} /(1+\mathrm{K})]=-2.301 / \lg [1 /(1+\mathrm{K})]$

в. дробная экстракция; в условиях экспериментов в противоточной колонне, величина $\left(\mathrm{W}_{2} / \mathrm{W}_{1}\right)$ равна 5.25 для экстракции СК $\mathrm{CO}_{2}$ при $10 \mathrm{MПа} \mathrm{и} 4.86$ для $\mathrm{CHF}_{3}$ при 7 МПа; в табл. 1 приведены величины $\mathrm{m}$, рассчитанные по уравнению (10) для этих случаев.

Расчёты показывают, что величины коэффициента межфазного распределения позволяют добиться требуемой степени очистки при умеренном расходе экстрагента даже в наименее благоприятном случае выделения $\Phi-3$ из водного раствора с использованием $\mathrm{CHF}_{3}$, если вести экстракцию в несколько стадий или дробно. При заданных параметрах работы противоточной колонны указанная степень очистки воды должна достигаться во всех рассмотренных случаях. При использовании в качестве экстрагента СК $\mathrm{CO}_{2}$ можно ожидать значительно более высоких степеней очистки водной фазы.

Действительно, приведённые в таблице 2 данные показывают, что концентрации Ф-3 в рафинате после проведения экстракции в противоточной колонне при различных условиях соответствует высоким степеням очистки, превышающим 0.995. При этом величины параметров эксперимента и величины К для экстрагента в указанных диапазонах варьирования не оказывают существенного влияния на эффективность выделения Ф-3 из водной фазы. Степень очистки несколько снижается при уменьшении времени контакта, что легко объясняется конечностью скорости массопереноса - перераспределения растворённого вещества между фазами. Даже при переходе от $\mathrm{CO}_{2}$ к $\mathrm{CHF}_{3}$ и снижении вдвое величины $\mathrm{K}$, степень очистки остаётся достаточно высокой, что говорит о высокой эффективности экстракции в противоточном режиме.

Таблица 2. Экстракция Ф-3 из нейтрального водного раствора $\left(3.99\right.$ г/дм $\left.{ }^{3}\right)$ при $50^{\circ} \mathrm{C}$; скорость подачи экстрагента 20 г/мин.

Table 2. Extraction of F-3 from a neutral aqueous solution $(3.99 \mathrm{~g} / \mathrm{l})$ at $50^{\circ} \mathrm{C}$; extractant feed rate 20 $\mathrm{g} / \mathrm{min}$

\begin{tabular}{|c|c|c|c|c|}
\hline № & $\begin{array}{c}\text { Порт подачи вод- } \\
\text { ного раствора }\end{array}$ & Экстрагент & $\begin{array}{c}\text { Концентрация в } \\
\text { рафинате, мг/дм }\end{array}$ & Степень очистки (Х) \\
\hline 1. & верхний & $\mathrm{CO}_{2}, 10 \mathrm{MПа}$ & 0.00045 & 0.9999 \\
\hline 2. & средний & $\mathrm{CO}_{2}, 10 \mathrm{MПа}$ & 0.011 & 0.9972 \\
\hline 3. & средний & $\mathrm{CHF}_{3}, 7 \mathrm{MПа}$ & 0.015 & 0.9962 \\
\hline
\end{tabular}

Таким образом, на основании величин коэффициентов межфазного распределения, полученных в статических условиях, предсказываются высокие степени очистки водных растворов от малорастворимых органических веществ. Результаты экспериментов в динамическом противоточном режиме показывают хорошее соответствие со сделанным прогнозом. При менее благоприятных соотношениях растворимостей органических веществ в водных системах и в СК флюидах повышение эффективности очистки возможно при одновременном увеличении количества экстрагента на единицу объёма водного раствора и эффективного значения величины $\mathrm{m}$. Первое достигается снижением скорости подачи водного раствора, а второе - пропорциональ- 
ным снижением скоростей подачи как водного раствора, так и экстрагента, либо увеличением длины зоны контакта фаз. Так, например, двукратное снижение скорости подачи водного раствора при двукратном увеличении физической высоты участка колонны, на котором происходит контакт фаз, может привести к росту степени очистки водного раствора от органического вещества с 0.9 до 0.995 при относительно низком значении K.

\section{Заключение}

Коэффициенты межфазного распределения для двух модельных объектов-ТБФ и Ф-3 были определены на основании полученных данных по экстракции в статических условиях между водной фазой и СК флюидами $-\mathrm{CO}_{2}$ и $\mathrm{CHF}_{3}$ при $50^{\circ} \mathrm{C}$ и плотности флюидов около 0.4 г $/ \mathrm{cm}^{3}$. Было установлено, что в условиях эксперимента СК $\mathrm{CO}_{2}$ проявил лучшую экстракционную способность из водных растворов по отношению к ТБФ и Ф-3, чем СК $\mathrm{CHF}_{3}$. Полученные величины К были использованы для оценки эффективности экстракционной очистки водных растворов от ТБФ и Ф-3 в различных режимах, а корректность оценки была продемонстрирована в экспериментах в противоточной экстракционной колонне (ПЭК). Показано, что во всех изученных парах «извлекаемый компонент-экстрагент» степень очистки в ПЭК может достигать 0.995 при умеренном расходе экстрагента.

\section{Работа выполнена при финансовой поддержке ГК «Росатом» в рамках проектного направления «Прорыв».}

\section{Список литературы}

1. Касаткин А.Г. Основные процессы и аппараты химической технологии. 2004. М. АльянС. 784 с.

2. Paulitis M.E., Penninger J.M., Gray R.D., Davison K.P. Chemical Engineering at Supercritical Fluid Conditions. Ann Arbor Science Publishers. MI. 1983. 207 p.

3. McHugh M., Krukonis V. Supercritical Fluid Extraction Principles and Practice. Butterworth-Heinemann, Boston. 1994. 366 p.

4. Brunner G. Gas Extraction an Introduction to Fundamentals of Supercritical Fluids and the Applications to Separation Processes. Steinkopff Darmstadt Springer. New York. 1994. pp. 59-86.

5. Taylor L. Supercritical Fluid Extraction. John Wiley\&Sons Inc. New York. 1996. 88 p.

6. Mukhopadhyay M. Natural Extracts Using Supercritical Carbon Dioxide. CRC Press LLC. Boca Raton. FL. 2000. pp. 22-34.

7. Brunner G. // J. of Supercritical Fluids. 2009. Vol. 47. pp. 574-582, DOI:10.1016/j.supflu.2008.09.022.

8. Menzio J., Binello A., Barge A., Cravotto G. // Processes. 2020. Vol. 8. pp. 1062, DOI:10.3390/pr8091062

9. Tello J., Viguera M., Calvo L. // J. of Supercritical Fluids. 2011. Vol. 59, pp. 53-60, DOI:10.1016/j.supflu.2011.07.018
10.Tsuda T., Mizuno K., Ohshima K., Kawakishi S. et al. // J. Agric. Food Chem. 1995. Vol. 43. pp. 2803-2806, DOI:10.1021/jf00059a006

11.Tena M.T., Valcárcel M., Hidalgo P., Ubera J.L. // Anal. Chem. 1997. Vol. 69. pp. 521-526. DOI:10.1021/ac960506t

12.Poiana M., Sicari V., Mincione B. // J. Essent. Oil Res. 1998. Vol. 10. pp. 145-152, Doi:10.1080/10412905.1998.9700866

13. Sargenti S.R., Lancüas F.M. // J. Chromatogr. Sci. 1998. Vol. 36. pp. 169-174, DOI:10.1093/chromsci/36.4.169

14.Señoráns F.J., Ruiz-Rodriguez A., Cavero S., Cifuentes A. et al. // J. Agric. Food Chem. 2001. Vol. 49. pp. 6039-6044, DOI:10.1021/jf010762t

15.Jakobsson M., Sivik B., Bergqvist P.A., Strandberg B. et al. // J. of Supercritical Fluids. 1994. Vol. 7. pp. 197-200, DOI: $10.1021 / \mathrm{jf0} 10762 \mathrm{t}$

16.Jan H.S., Wang W.Ch. // Separation and Purification Technology. 2020. Vol. 242. p. 116781, Doi:10.1016/j.seppur.2020.116781

17.Señoráns F.J., Ruiz-Rodriguez A., Ibañez E., Tabera J. et al. // J. Supercrit. Fluids. 2001. Vol. 21. pp. 41-49. 
18.Bejarano A., del Valle J.M. // J. Supercritical Fluids. 2017. Vol. 120/2. pp. 266274. Doi:10.1016/j.supflu.2016.08.001.

19.Terada A., Kitajima N., Machmudah S., Tanaka M. et al. // Sep. Purif. Technol. 2010. Vol. 71 (1). pp. 107-113.

20. Химия ядерного горючего. М. Госхимиздат. $1956.552 \mathrm{c}$.

21.Золотов Ю.А., Спиваков Б.Я., Химия процессов экстракции. 1972. М. Наука. 288 с.

22.Кольдиц Л. Анорганикум. Т. 1. М. 1984. Мир. 672 c.
23.Айнштейн В.Г. Общий курс процессов и аппаратов химической технологии. ч. 2. 2002. М. Химия. 2002. 872 с.

24.Velavendan P., Ganesh S., Pandey N.K., Geetha R. et al. // J. Radioanal. Nucl. Chem. 2013. Vol. 295. pp. 1113-1117. Doi:10.1007/s10967-012-1945-1

25.NIST Chemistry WebBook, NIST Standard Reference Database Number 69. 2018 (https://webbook.nist.gov/chemistry/);

DOI:10.18434/T4D303.

\title{
Evaluation of the efficiency of extraction of poorly soluble impurities from aqueous solutions by supercritical fluids in dynamic flow mode using chromatography-mass spectrometry
}

\author{
(C) 2020 Parenago O.O. ${ }^{1,2}$, Kostenko M.O. ${ }^{1}$, Ustinovich K.B. ${ }^{1}$, \\ Khesina Z.B. ${ }^{3}$, Buryak A.K. ${ }^{3}$ \\ ${ }^{1}$ Institute of General and inorganic chemistry of the Russian Academy of Sciences, Moscow \\ ${ }^{2}$ Chemistry Department of Lomonosov MSU, Lenin hills, Moscow \\ ${ }^{3}$ Russia A.N. Frumkin Institute of Physical Chemistry and Electrochemistry, Moscow
}

\begin{abstract}
Supercritical fluid extraction (SFE) is actively used in the separation of valuable components or especially toxic and radioactive substances from various natural sources, such as water systems. Despite the active study of this method, its possibilities have not yet been fully studied. In this study, two low-soluble substances, tributyl phosphate (TBP) and methanitrobenzotrifluoride (meta-nitro-trifluoromethyl-benzene, F-3), were used for the release of radionuclides during the reprocessing of spent nuclear fuel (SNF) were considered as model objects. The purpose of this study was the evaluation of the efficiency of the extraction purification of aqueous solutions containing TBP and F-3 under dynamic conditions based on the estimated calculation and the verification of its results in an experiment on a counter-current extraction column. The calculation was based on the data on the distribution of impurities between the water and fluid phases, which were obtained in static experiments. The concentrations of substances in the initial solutions (before extraction) were determined by gas chromatography with mass spectrometric detection (GC-MS) using a SHIMADZU GCMS-TQ8040 instrument. For chromatographic separation, a standard ZB-5MS capillary column $(60 \mathrm{~m} \times 0.25 \mathrm{~mm} \times 0.25 \mu \mathrm{m})$ was used. The same method was applied for the determination of the residual concentrations of TPB and F-3 in aqueous solutions after extraction. Based on the extraction data under static conditions, the interfacial distribution coefficients $(\mathrm{K})$ were determined for TBP and F-3 between the aqueous phase and supercritical (SC) fluids - $\mathrm{CO}_{2}$ and R-23 Freon $\left(\mathrm{CHF}_{3}\right)$ at $50^{\circ} \mathrm{C}$ with a fluid density of about $0.4 \mathrm{~g} / \mathrm{cm}^{3}$. It was shown that under these conditions $\mathrm{SC} \mathrm{CO}_{2}$ is a more effective extractant for the isolation of TBP and F-3 from aqueous solutions. The obtained $\mathrm{K}$ values were used to evaluate the efficiency of the extraction purification of aqueous solutions from TBP and F-3 in various modes. The performance of such an evaluation has been demonstrated in experiments in a counter-current extraction column (PEC). It is shown that in all the studied pairs of "extractable component - extractant", the degree of purification in PEC can reach 0.995 with moderate consumption of the extractant.
\end{abstract}

Keywords: interfacial distribution coefficient, supercritical fluid, counter-current extraction column.

\section{References}

1. Kasatkin A.G., Osnovnye processy i apparaty khimicheskoj tekhnologii, M., Al'janS, 2004, $784 \mathrm{p}$
2. Paulitis M.E., Penninger J.M., Gray R.D., Davison K.P. Chemical Engineering at Supercritical Fluid Conditions. Ann Arbor Science Publishers, MI, 1983, 207 p. 
3. McHugh M., Krukonis V., Supercritical Fluid Extraction Principles and Practice. Butterworth-Heinemann, Boston, 1994. 366 p.

4. Brunner G. Gas Extraction an Introduction to Fundamentals of Supercritical Fluids and the Applications to Separation Processes. Steinkopff Darmstadt Springer, New York, 1994. pp. 59-86.

5. Taylor L. Supercritical Fluid Extraction. John Wiley\&Sons Inc, New York, 1996. 88 p.

6. Mukhopadhyay M. Natural Extracts Using Supercritical Carbon Dioxide, CRC Press LLC, Boca Raton, FL, 2000. pp. 22-34.

7. Brunner G., J. of Supercritical Fluids, 2009, Vol. 47, pp. 574-582, Doi:10.1016/j.supflu.2008.09.022.

8. Menzio J., Binello A., Barge A., Cravotto G., Processes, 2020, Vol. 8, p. 1062, Doi:10.3390/pr8091062

9. Tello J., Viguera M., Calvo L., J. of Supercritical Fluids, 2011, Vol. 59, pp. 53-60, Doi:10.1016/j.supflu.2011.07.018

10. Tsuda T., Mizuno K., Ohshima K., Kawakishi S. et al., J. Agric. Food Chem., 1995, Vol. 43, pp. 2803-2806, Doi:10.1021/jf00059a006

11. Tena M.T., Valcárcel M., Hidalgo P., Ubera J.L., Anal. Chem. 1997, Vol. 69, pp. 521526. Doi:10.1021/ac960506t

12. Poiana M., Sicari V., Mincione B., J. Essent. Oil Res., 1998, Vol. 10, pp. 145-152, Doi:10.1080/10412905.1998.9700866

13. Sargenti S.R., Lancüas F.M., $J$. Chromatogr. Sci., 1998, Vol. 36, pp. 169-174, Doi:10.1093/chromsci/36.4.169

14. Señoráns F.J., Ruiz-Rodriguez A., Cavero S., Cifuentes A., et al., J. Agric. Food Chem.,

Паренаго Ольга Олеговна - к.х.н., старший научный сотрудник Института общей и неорганической химии им. Н.С. Курнакова Российской Академии Наук (ИОНХ РАН), Химический факультет МГУ им. М.В. Ломоносова, Москва

Костенко Михаил Олегович - младший научный сотрудник Института общей и неорганической химии им. Н.С. Курнакова Российской Академии Наук (ИОНХ РАН), Москва

Устинович Константин Борисович - инженертехнолог Института общей и неорганической химии им. Н.С. Курнакова Российской Академии Наук (ИОНХ РАН), Москва

Хесина Зоя Борисовна - инженер-исследователь Института физической химии и электрохимии им. Фрумкина Российской Академии Наук (ИФХЭ РАН), Москва

Буряк Алексей Константинович - член-корреспондент РАН, д.Х.н., директор Института физической химии и электрохимии им. Фрумкина Российской Академии Наук, Москва
2001, Vol. 49, pp. 6039-6044.

Doi:10.1021/jf010762t

15. Jakobsson M., Sivik B., Bergqvist P.A., Strandberg B. et al., J. of Supercritical Fluids, 1994, Vol. 7, pp. 197-200, Doi:10.1021/jf010762t

16. Jan H.S., Wang W.Ch., Separation and Purification Technolog, 2020, Vol. 242, p. 116781, Doi:10.1016/j.seppur.2020.116781

17. Señoráns F.J., Ruiz-Rodriguez A., Ibañez E., Tabera J. et al., J. Supercrit. Fluids, 2001, Vol. 21, pp. 41-49.

18. Bejarano A., del Valle J.M., J. Supercritical Fluids, 2017, Vol. 120/2, pp. 266274. Doi:10.1016/j.supflu.2016.08.001.

19. Terada A., Kitajima N., Machmudah S., Tanaka M. et al., Sep. Purif. Technol., 2010, Vol. 71 (1), pp. 107-113.

20. Khimija jadernogo gorjuchego. M., Goshimizdat, 1956, $552 \mathrm{p}$.

21. Zolotov Ju.A., Spivakov B.Ja., Khimija processov jekstrakcii, M., Nauka, 1972, 288 p.

22. Kol'dic L., Anorganikum, Vol. 1, M., Mir, 1984, 672 p.

23. Ajnshtejn V.G., Obshhij kurs processov i apparatov himicheskoj tehnologii, ch.2, Moskva, Khimija, 2002, 872 p.

24. Velavendan P., Ganesh S., Pandey N.K., Geetha R. et al., J. Radioanal. Nucl. Chem., 2013, Vol. 295, pp. 1113-1117. Doi:10.1007/s10967-012-1945-1.

25. NIST Chemistry WebBook, NIST Standard Reference Database Number 69, 2018 (https://webbook.nist.gov/chemistry/);

DOI:10.18434/T4D303.

Parenago Olga O. - PhD, senior researcher, Kurnakov Institute of General and Inorganic Chemistry Russian Academy of Sciences (IGIC RAS), Chemistry Department of Lomonosov MSU, Moscow, e-mail: oparenago@mail.ru

Kostenko Mikhail O. - junior researcher, Kurnakov Institute of General and Inorganic Chemistry Russian Academy of Sciences (IGIC RAS), Moscow, e-mail: kostenko@supercritical.ru

Ustinovich Konstantin B. - engineer-technologist, Kurnakov Institute of General and Inorganic Chemistry Russian Academy of Sciences (IGIC RAS), Moscow, email: ustinovich@supercritical.ru

Khesina Zoya B. - research engineer, A.N. Frumkin Institute of Physical chemistry and Electrochemistry Russian academy of sciences (IPCE RAS), Moscow, email: maldi-ms@yandex.ru

Buryak Alexey K. - Doctor of science (chemistry), professor, A.N. Frumkin Institute of Physical chemistry and Electrochemistry Russian academy of sciences (IPCE RAS), Moscow, e-mail: akburyak@yandex.ru 